\title{
TRIMMING OF METRIC SPACES AND THE TIGHT SPAN
}

\author{
VLADIMIR TURAEV
}

\begin{abstract}
We use the trimming transformations to study the tight span of a metric space.
\end{abstract}

\section{INTRODUCTION}

The theory of tight spans due to J. Isbell Is and A. Dress Dr embeds any metric space $X$ in a hyperconvex metric space $T(X)$ called the tight span of $X$. In this paper we split $T(X)$ as a union of two metric subspaces $\tau=\tau(X)$ and $\bar{C}=\overline{C(X)}$. The space $\tau$ is the tight span of a certain quotient $\overline{X_{\infty}}$ of $X$. The space $\bar{C}$ is a disjoint union of metric trees which either do not meet $\tau$ or meet $\tau$ at their roots lying in $\overline{X_{\infty}} \subset \tau$. In this picture, the original metric space $X \subset T(X)$ consists of the tips of the branches of the trees.

The construction of $\tau$ and $\bar{C}$ uses the trimming transformations of metric spaces studied in $\mathrm{Tu}$ for finite metric spaces. In the present paper - essentially independent of $[\mathrm{Tu}$ - we discuss trimming for all metric spaces and introduce related objects including the subspaces $\tau$ and $\bar{C}$ of $T(X)$. Our main theorem says that $\tau \cup \bar{C}=T(X)$ and $\tau \cap \bar{C}$ is the set of the roots of the trees forming $\bar{C}$.

This work was partially supported by the NSF grant DMS-1664358.

\section{TRIM PSEUdOMETRIC SPACES}

2.1. Pseudometrics. We recall basics on metric and pseudometric spaces. Set $\mathbb{R}_{+}=\{r \in \mathbb{R} \mid r \geq 0\}$. A pseudometric space is a pair consisting of a set $X$ and a mapping $d: X \times X \rightarrow \mathbb{R}_{+}$(the pseudometric) such that for all $x, y, z \in X$,

$$
d(x, x)=0, \quad d(x, y)=d(y, x), \quad d(x, y)+d(y, z) \geq d(x, z) .
$$

A pseudometric space $(X, d)$ is a metric space (and $d$ is a metric) if $d(x, y)>0$ for all distinct $x, y \in X$.

A map $f: X \rightarrow X^{\prime}$ between pseudometric spaces $(X, d)$ and $\left(X^{\prime}, d^{\prime}\right)$ is distance preserving if $d(x, y)=d^{\prime}(f(x), f(y))$ for all $x, y \in X$ and is non-expansive if $d(x, y) \geq d^{\prime}(f(x), f(y))$ for all $x, y \in X$. Pseudometric spaces $X, X^{\prime}$ are isometric if there is a distance preserving bijection $X \rightarrow X^{\prime}$. We call distance preserving maps between metric spaces metric embeddings; they are always injective.

Each pseudometric space $(X, d)$ carries an equivalence relation $\sim_{d}$ defined by $x \sim_{d} y$ if $d(x, y)=0$ for $x, y \in X$. The quotient set $\bar{X}=X / \sim_{d}$ carries a metric $\bar{d}$ defined by $\bar{d}(\bar{x}, \bar{y})=d(x, y)$ where $x, y$ are any points of $X$ and $\bar{x}, \bar{y} \in \bar{X}$ are their 
equivalence classes. The metric space $(\bar{X}, \bar{d})$ is the metric quotient of $(X, d)$. Any distance preserving map from $X$ to a metric space $Y$ expands uniquely as the composition of the projection $X \rightarrow \bar{X}$ and a metric embedding $\bar{X} \hookrightarrow Y$.

2.2. Trim spaces. Given a set $X$ and a map $d: X \times X \rightarrow \mathbb{R}$, we use the same symbol $d$ for the map $X \times X \times X \rightarrow \mathbb{R}$ carrying any triple $x, y, z \in X$ to

$$
d(x, y, z)=\frac{d(x, y)+d(x, z)-d(y, z)}{2} .
$$

The right-hand side is called the Gromov product, see, for instance, $\mathrm{BCK}$.

A pseudometric $d$ in a set $X$ determines a function $\underline{d}: X \rightarrow \mathbb{R}_{+}$as follows: if $\operatorname{card}(X)=1$, then $\underline{d}=0$; if $X$ has two points $x, y$, then $\underline{d}(x)=\underline{d}(y)=d(x, y) / 2$; if $\operatorname{card}(X) \geq 3$, then for all $x \in X$,

$$
\underline{d}(x)=\inf _{y, z \in X \backslash\{x\}, y \neq z} d(x, y, z) .
$$

If $\underline{d}(x)=0$ for all $x \in X$, then we say that the pseudometric space $(X, d)$ is trim.

Following $\mathrm{K}$. Menger $\mathrm{Me}$, we say that a point $x$ of a pseudometric space $(X, d)$ lies between $y \in X$ and $z \in X$ if $d(y, z)=d(x, y)+d(x, z)$. A simple sufficient condition for $(X, d)$ to be trim says that each point $x \in X$ lies between two distinct points of $X \backslash\{x\}$.

2.3. Examples. A pseudometric space having only one point is trim. A pseudometric space having two points is trim iff the distance between these points is equal to zero. More generally, any set with zero pseudometric is trim. We give two examples of trim metric spaces:

(a) the set of words of a fixed finite length in a given finite alphabet with the Hamming distance between words defined as the number of positions at which the letters of the words differ;

(b) a subset of a Euclidean circle $C \subset \mathbb{R}^{2}$ meeting each half-circle in $C$ in at least three points; here the distance between two points is the length of the shorter arc in $C$ connecting these points.

\section{DRIFT AND TRIMMING}

3.1. The drift. Given a function $\delta: X \rightarrow \mathbb{R}$ on a metric space $(X, d)$, we define a map $d_{\delta}: X \times X \rightarrow \mathbb{R}$ as follows: for $x, y \in X$,

$$
d_{\delta}(x, y)=\left\{\begin{array}{l}
0 \quad \text { if } x=y, \\
d(x, y)-\delta(x)-\delta(y) \quad \text { if } x \neq y .
\end{array}\right.
$$

We say that $d_{\delta}$ is obtained from $d$ by a drift. The idea behind the definition of $d_{\delta}$ is that each point $x \in X$ is pulled towards all other points of $X$ by $\delta(x)$.

Lemma 3.1. If $\delta \leq \underline{d}$ (i.e., if $\delta(x) \leq \underline{d}(x)$ for all $x \in X$ ), then $d_{\delta}$ is a pseudometric in $X$. Moreover, if $\operatorname{card}(X) \geq 3$ or $\operatorname{card}(X)=2, \delta=$ const, then $\underline{d_{\delta}}=\underline{d}-\delta$.

Proof. We first prove that for any distinct $x, y \in X$,

$$
\underline{d}(x)+\underline{d}(y) \leq d(x, y) .
$$


If $X=\{x, y\}$, then (3.1.1) follows from the definition of $\underline{d}$. If $\operatorname{card}(X) \geq 3$, pick any $z \in X \backslash\{x, y\}$. Then $\underline{d}(x) \leq d(x, y, z)$ and $\underline{d}(y) \leq d(y, x, z)$. So,

$$
\underline{d}(x)+\underline{d}(y) \leq d(x, y, z)+d(y, x, z)=d(x, y) .
$$

We now check that $d_{\delta}: X \times X \rightarrow \mathbb{R}$ is a pseudometric. Clearly, $d_{\delta}$ is symmetric and, by definition, $d_{\delta}(x, x)=0$ for all $x \in X$. Formula (3.1.1) and the assumption $\delta \leq \underline{d}$ imply that for any distinct $x, y \in X$,

$$
d_{\delta}(x, y)=d(x, y)-\delta(x)-\delta(y) \geq \underline{d}(x)-\delta(x)+\underline{d}(y)-\delta(y) \geq 0 .
$$

To prove the triangle inequality for $d_{\delta}$ we rewrite it as $d_{\delta}(x, y, z) \geq 0$ for any $x, y, z \in X$. If $x=y$ or $x=z$, then $d_{\delta}(x, y, z)=0$; if $y=z$, then $d_{\delta}(x, y, z)=$ $d_{\delta}(x, y) \geq 0$. Finally, if $x, y, z$ are pairwise distinct, then

$$
d_{\delta}(x, y, z)=d(x, y, z)-\delta(x) \geq \underline{d}(x)-\delta(x) \geq 0 .
$$

The equality $\underline{d_{\delta}}(x)=\underline{d}(x)-\delta(x)$ for all $x \in X$ follows from (3.1.2) if $\operatorname{card}(X) \geq$ 3 and from the definitions if $\operatorname{card}(X)=2$ and $\delta=$ const.

3.2. Trimming. Let $(X, d)$ be a metric space. Applying Lemma 3.1 to $\delta=\underline{d}$ : $X \rightarrow \mathbb{R}$, we obtain a pseudometric $d^{-}=d_{\delta}$ in $X$. For $x, y \in X$,

$$
d^{-}(x, y)=\left\{\begin{array}{l}
0 \quad \text { if } x=y, \\
d(x, y)-\underline{d}(x)-\underline{d}(y) \quad \text { if } x \neq y .
\end{array}\right.
$$

Lemma 3.1 implies that $\underline{d^{-}}=0$, i.e., that $\left(X, d^{-}\right)$is a trim pseudometric space. Let $t(X)$ be the metric quotient of $\left(X, d^{-}\right)$. By definition, $t(X)=X / \sim_{d^{-}}$and the metric in $t(X)$ is induced by the pseudometric $d^{-}$in $X$. We say that $t(X)$ is obtained from $(X, d)$ by trimming and call the projection $p_{X}: X \rightarrow t(X)$ the trimming projection. Note that $p_{X}$ is a non-expansive surjection of metric spaces. It is bijective if and only if $d^{-}$is a metric in $X$ and then $t(X)=\left(X, d^{-}\right)$is a trim metric space. In general, $t(X)$ may be non-trim.

Starting from $\left(X_{0}, d_{0}\right)=(X, d)$ and iterating the trimming, we obtain metric spaces $\left\{t^{k}(X)=\left(X_{k}, d_{k}\right)\right\}_{k \geq 1}$ and non-expansive surjections

$$
X=X_{0} \stackrel{p_{0}}{\longrightarrow} X_{1} \stackrel{p_{1}}{\longrightarrow} X_{2} \stackrel{p_{2}}{\longrightarrow} \cdots
$$

where $p_{0}=p_{X}$ and $p_{k}=p_{t^{k}(X)}: X_{k} \rightarrow X_{k+1}$ for $k \geq 1$. We call the sequence (3.2.2) the trimming sequence of $X$. Each point $x \in X$ determines points $\left(x_{(k)} \in\right.$ $\left.X_{k}\right)_{k \geq 0}$ by $x_{(0)}=x$ and $x_{(k+1)}=p_{k}\left(x_{(k)}\right)$ for all $k$. The sequence $\left(x_{(k)}\right)_{k \geq 0}$ is the trimming sequence of $x$.

For $x, y \in X$, we write $x \cong y$ if there is an integer $k \geq 0$ such that $x_{(k)}=y_{(k)}$. We call the smallest such $k$ the meeting index of $x, y$ and denote it by $m(x, y)$. Note that $x_{(k)}=y_{(k)}$ for all $k \geq m(x, y)$ which easily implies that $\cong$ is an equivalence relation in $X$. Clearly, $m(x, x)=0$ and $m(x, y) \geq 1$ for $x \neq y$.

Set $X_{\infty}=X / \cong$ and for $x \in X$, denote its projection to $X_{\infty}$ by $x_{(\infty)}$. We have

$$
d(x, y) \geq d_{1}\left(x_{(1)}, y_{(1)}\right) \geq d_{2}\left(x_{(2)}, y_{(2)}\right) \geq \cdots \geq 0
$$

for any $x, y \in X$. As a consequence, the formula

$$
d_{\infty}\left(x_{(\infty)}, y_{(\infty)}\right)=\lim _{k \rightarrow \infty} d_{k}\left(x_{(k)}, y_{(k)}\right)
$$


defines a pseudometric $d_{\infty}$ in $X_{\infty}$. Clearly, the projection $X \rightarrow X_{\infty}, x \mapsto x_{(\infty)}$ is a non-expansive surjection.

3.3. Remark. One can take the metric quotient $\overline{X_{\infty}}$ of $X_{\infty}$ and then iterate the construction $X \mapsto \overline{X_{\infty}}$. We will not do it in this paper.

\section{LeAF SPACES OF METRIC TREes}

We compute the trimming for the leaf spaces of metric trees.

4.1. Metric graphs and trees. A metric graph is a graph without loops whose every edge $e$ is endowed with a real number $\ell_{e}>0$, the length, and with a homeomorphism $e \approx\left[0, \ell_{e}\right] \subset \mathbb{R}_{+}$. We use this homeomorphism to define the length of any subsegment of $e$ : this is just the length of the corresponding subsegment of $\left[0, \ell_{e}\right]$. A wider class of pseudometric graphs is defined similarly but allowing the lengths of edges to be equal to zero. Only the edges of positive length carry a homeomorphism $e \approx\left[0, \ell_{e}\right]$. All subsegments of edges of zero length have zero length.

A pseudometric (respectively, metric) tree is a pseudometric (respectively, metric) graph which is path connected and has no cycles. The underlying set of a pseudometric tree $L$ carries a path pseudometric $d_{L}$ : the distance $d_{L}(x, y)$ between any points $x, y \in L$ is the sum of the lengths of the edges and subedges forming an injective path from $x$ to $y$ in $L$. The pseudometric $d_{L}$ is a metric if and only if $L$ is a metric tree. Every pseudometric tree $L$ yields a metric tree $\bar{L}$ by contracting each edge of $L$ of zero length to a point while keeping the homeomorphisms associated with the edges of positive length. The underlying metric space $\left(\bar{L}, d_{\bar{L}}\right)$ of $\bar{L}$ is nothing but the metric quotient of $\left(L, d_{L}\right)$.

4.2. The leaf space. A leaf of a pseudometric tree $L$ is a vertex of $L$ adjacent to a single edge of $L$. The leaf space $\partial L$ is the set of all leaves of $L$ together with the pseudometric $d$ obtained by restricting $d_{L}$ to this set. The pseudometric space $(\partial L, d)$ depends only on the tree $L$ and the lengths of its edges; it does not depend on the choice of homeomorphisms $e \approx\left[0, \ell_{e}\right]$. The following lemma estimates (and in some cases computes) the function $\underline{d}: \partial L \rightarrow \mathbb{R}_{+}$.

Lemma 4.1. Let $(\partial L, d)$ be the leaf space of a pseudometric tree $L$ such that $\operatorname{card}(\partial L) \geq 3$. Then for all $x \in \partial L$, we have $\underline{d}(x) \geq l_{e(x)}$ where $e(x)$ is the edge of $L$ adjacent to $x$. If the second vertex of $e(x)$ is adjacent to at least two leaves of $L$ besides $x$, then $\underline{d}(x)=l_{e(x)}$.

Proof. Let $v$ be the vertex of the edge $e(x)$ distinct from $x$. Pick any $y, z \in$ $\partial L \backslash\{x\}$. It is clear that an injective path from $x$ to $y$ passes through $v$. Therefore $d_{L}(x, y)=l_{e(x)}+d_{L}(v, y)$ and, similarly, $d_{L}(x, z)=l_{e(x)}+d_{L}(v, z)$. Then

$$
\begin{gathered}
d(x, y, z)=\frac{d_{L}(x, y)+d_{L}(x, z)-d_{L}(y, z)}{2} \\
=l_{e(x)}+\frac{d_{L}(v, y)+d_{L}(v, z)-d_{L}(y, z)}{2} \geq l_{e(x)} .
\end{gathered}
$$


Since this holds for all $y, z$ as above, $\underline{d}(x) \geq l_{e(x)}$. Suppose now that $v$ is adjacent to distinct leaves $y, z \in \partial L \backslash\{x\}$. Then the edges $e(x), e(y)$ form an injective path from $x$ to $y$ and so $d_{L}(x, y)=l_{e(x)}+l_{e(y)}$. Similarly, $d_{L}(x, z)=l_{e(x)}+l_{e(x)}$ and $d_{L}(y, z)=l_{e(y)}+l_{e(z)}$. Then $d(x, y, z)=l_{e(x)}$. Therefore $\underline{d}(x)=l_{e(x)}$.

4.3. Example. Let $L$ be a metric tree whose every vertex adjacent to a leaf is adjacent to at least three leaves. Lemma 4.1 implies that $t(\partial L)$ is the set of all vertices of $L$ adjacent to a leaf with the metric $d_{L}$ restricted to this set.

4.4. Example. Consider an infinite sequence of sets and surjective maps

$$
X_{0} \stackrel{p_{0}}{\longrightarrow} X_{1} \stackrel{p_{1}}{\longrightarrow} X_{2} \stackrel{p_{2}}{\longrightarrow} \cdots .
$$

Fix any functions $\left\{\delta_{k}: X_{k} \rightarrow(0, \infty)\right\}_{k \geq 0}$. Consider the metric graph $L$ with the set of vertices $\amalg_{k \geq 0} X_{k}$ and with each $v \in X_{k}$ connected to $p_{k}(v) \in X_{k+1}$ by an edge of length $\delta_{k}(v)$. Assume that $\operatorname{card}\left(p_{k}^{-1}(a)\right) \geq 3$ for all $k \geq 0$ and $a \in X_{k+1}$ and that for any distinct $x, y \in X$ there is an integer $m=m(x, y) \geq 1$ such that

$$
\left(p_{m} \cdots p_{1} p_{0}\right)(x)=\left(p_{m} \cdots p_{1} p_{0}\right)(y) .
$$

Then $L$ is a metric tree. For all $k \geq 0$, restricting the tree metric $d_{L}$ to $X_{k} \subset L$, we obtain a metric space $\left(X_{k}, d_{k}\right)$ where $d_{k}$ is computed by

$$
d_{k}(x, y)=\sum_{i=k}^{m-1}\left(\delta_{i}\left(\left(p_{i-1} \cdots p_{k}\right)(x)\right)+\delta_{i}\left(\left(p_{i-1} \cdots p_{k}\right)(y)\right)\right)
$$

for any distinct $x, y \in X_{k}$, where $m>k$ is the smallest integer such that

$$
\left(p_{m-1} \cdots p_{k}\right)(x)=\left(p_{m-1} \cdots p_{k}\right)(y) .
$$

Lemma 4.1 implies that $d_{k}=\delta_{k}$ and $\left(X_{k+1}, d_{k+1}\right)$ is obtained from $\left(X_{k}, d_{k}\right)$ by trimming: $\left(X_{k+1}, d_{k+1}\right)=t\left(X_{k}, d_{k}\right)$. Then the sequence (4.4.1) is the trimming sequence of $\partial L=\left(X_{0}, d_{0}\right)$ and $X_{\infty}$ is a singleton (i.e., $\operatorname{card}\left(X_{\infty}\right)=1$ ).

4.5. Example. We describe sets and maps satisfying the conditions of Example 4.4. Let $A$ be a set with $\geq 3$ elements and let $a \in A$. For $k \geq 0$, let $X_{k}$ be the set of all infinite sequences $a_{0}, a_{1}, \ldots$ of elements of $A$ such that $a_{n}=a$ for all sufficiently big $n$. The map $p_{k}: X_{k} \rightarrow X_{k+1}$ drops the first element of a sequence. Given functions $\left\{\delta_{k}: X_{k} \rightarrow(0, \infty)\right\}_{k \geq 0}$, Example 4.4 provides, for each $k \geq 0$, a metric $d_{k}$ in $X_{k}$ such that $\left(X_{k+1}, d_{k+1}\right)=t\left(X_{k}, d_{k}\right)$ for all $k$.

\section{The TRIMMing CYLINDER}

We introduce the trimming cylinder of a metric space. 
5.1. The cylinder $C$. Consider a metric space $(X, d)=\left(X_{0}, d_{0}\right)$ and its trimming sequence (3.2.2) where $t^{k}(X)=\left(X_{k}, d_{k}\right)$ for all $k \geq 1$. We define a graph $C=C(X)$ : take the disjoint union $\amalg_{k \geq 0} X_{k}$ as the set of vertices and connect each $v \in X_{k}$ to $p_{k}(v) \in X_{k+1}$ by an edge $e_{v}$ of length $d_{k}(v) \geq 0$. If $d_{k}(v)>0$, then we take for $e_{v}$ a copy of the segment $\left[0, \underline{d_{k}}(v)\right] \subset \mathbb{R}_{+}$. Clearly, $C$ is a pseudometric graph and $X=X_{0}$ is the set of its leaves. We call $C$ the trimming cylinder of $X$.

It is clear that all (path connected) components of $C$ are trees. Two points $x, y \in X \subset C$ lie in the same component of $C$ if and only if $x \cong y$. We can therefore identify the set $\pi_{0}(C)$ of components of $C$ with $X_{\infty}=X / \cong$.

Each component of $C$, being a pseudometric tree, carries the path pseudometric. Also, the set $X_{k} \subset C$ carries the metric $d_{k}$ for all $k \geq 0$. The following theorem extends all these pseudometrics and metrics to a pseudometric in $C$.

Theorem 5.1. There is a unique pseudometric $\rho$ in $C$ which restricts to the metric $d$ in $X \subset C$, restricts to the path pseudometric in every component of $C$, and is minimal in the class of such pseudometrics in $C$. For all $k \geq 1$, we have $\left.\rho\right|_{X_{k}}=d_{k}$.

The minimality of $\rho$ means that for any pseudometric $\rho^{\prime}$ in $C$ which restricts to $d$ in $X$ and to the path pseudometrics in the components of $C$, we have $\rho(a, b) \leq$ $\rho^{\prime}(a, b)$ for all $a, b \in C$. The uniqueness of such $\rho$ is obvious; we only need to prove the existence and the equality $\left.\rho\right|_{X_{k}}=d_{k}$. We do it in Section 6 .

In the proof of Theorem 5.1 we will use a partial order in $C$ defined as follows. We say that a path in $C$ goes down if whenever it enters an edge connecting a point of $X_{k}$ to a point of $X_{k+1}$, it goes in this edge in the direction from $X_{k}$ to $X_{k+1}$. We say that a point $b \in C$ lies below a point $a \in C$ if $a \neq b$ and there is a path in $C$ going down from $a$ to $b$. For example, for $x \in X$ and integers $k<l$, the point $x_{(l)} \in X_{l}$ lies below $x_{(k)} \in X_{k}$.

5.2. The metric space $\bar{C}$. Consider now the metric quotient $\bar{C}=(\bar{C}, \bar{\rho})$ of the trimming cylinder $(C=C(X), \rho)$. Since $X$ is a metric space, the embedding $X=X_{0} \subset C$ induces a metric embedding $X \hookrightarrow \bar{C}$. We view $X$ as a subspace of $\bar{C}$ via this embedding.

We say that a vertex $x_{(k)} \in X_{k}$ of $C$ is special if for all $l \geq k$, the edge of $C$ connecting $x_{(l)}$ to $x_{(l+1)}$ has zero length, i.e., $\underline{d}_{l}\left(x_{(l)}\right)=0$. A component $L$ of $C$ is special if it has at least one special vertex. Then all special vertices of $L$ and all edges between them form a tree which collapses into a single vertex in $\bar{L} \subset \bar{C}$. We call the latter vertex the root of $\bar{L}$.

We can now describe $\bar{C}$. If $\operatorname{card}\left(X_{\infty}\right)=1$, then $(C, \rho)$ is a pseudometric tree with the path pseudometric and $(\bar{C}, \bar{\rho})$ is the associated metric tree with the path metric. If $\operatorname{card}\left(X_{\infty}\right) \geq 2$, then $(\bar{C}, \bar{\rho})$ is obtained from $(C, \rho)$ in two steps. First, each component $L$ of $C$ is replaced with its metric quotient - the metric tree $\bar{L}$ (which has a distinguished root if $L$ is special). Second, for all $u, v \in X_{\infty}=\pi_{0}(C)$ such that $d_{\infty}(u, v)=0$ and both corresponding components $L_{u}, L_{v}$ of $C$ are special, we identify (glue) the roots of $\overline{L_{u}}$ and $\overline{L_{v}}$. This gives a metric graph whose components are trees. We endow this graph with the unique 
metric such that the projection from $C$ to this graph is distance preserving. The resulting metric space is $(\bar{C}, \bar{\rho})$ as is clear from the definition of $\rho$ in Section 6.2

5.3. Example. The trimming cylinders arising in Examples 4.4 and 4.5 have no special vertices. If we modify the assumptions there to $\delta_{k}\left(X_{k}\right) \subset(0, \infty)$ for $k=$ $0,1, \ldots, N$ with some $N \geq 0$ and $\delta_{k}=0$ for all $k>N$, then the trimming sequence of the metric space $\left(X_{0}, d_{0}\right)$ consists of the metric spaces $\left\{\left(X_{k}, d_{k}\right)\right\}_{k=0}^{N}$, the set $X_{N+1}$ with zero pseudometric, and singletons corresponding to all $k>N+1$. All the vertices of the associated trimming cylinder belonging to $X_{N+1}$ are special.

\section{The Series $\sigma$ And Proof of Theorem 5.1}

In this section, as above, $X=(X, d)$ is a metric space.

6.1. The series $\sigma$. For each $x \in X$, consider the infinite series

$$
\sigma(x)=\sum_{k=0}^{\infty} \underline{d_{k}}\left(x_{(k)}\right)=\underline{d}(x)+\sum_{k=1}^{\infty} \underline{d_{k}}\left(x_{(k)}\right) .
$$

Here $\left(x_{(k)} \in X_{k}\right)_{k \geq 0}$ is the trimming sequence of $x$ and $\underline{d_{k}}: X_{k} \rightarrow \mathbb{R}_{+}$is the function induced by the metric $d_{k}$ in $X_{k}$. For any $n \geq 1$, set

$$
\sigma^{n}(x)=\sum_{k=0}^{n-1} \underline{d_{k}}\left(x_{(k)}\right)=\underline{d}(x)+\sum_{k=1}^{n-1} \underline{d_{k}}\left(x_{(k)}\right) .
$$

This gives a well-defined function $\sigma^{n}: X \rightarrow \mathbb{R}_{+}$. In particular, $\sigma^{1}=\underline{d}$.

Lemma 6.1. (i) For any distinct $x, y \in X$ such that $x \cong y$, we have

$$
d(x, y)=\sigma^{m}(x)+\sigma^{m}(y)
$$

where $m=m(x, y) \geq 1$ is the meeting index of $x$ and $y$;

(ii) For any $x, y \in X$ such that $x \neq y$, both series $\sigma(x)$ and $\sigma(y)$ converge and

$$
d(x, y)=d_{\infty}\left(x_{(\infty)}, y_{(\infty)}\right)+\sigma(x)+\sigma(y) .
$$

Proof. To prove (i), note that for all $s<m$, we have $x_{(s)} \neq y_{(s)}$ and therefore

$$
d_{s}\left(x_{(s)}, y_{(s)}\right)-d_{s+1}\left(x_{(s+1)}, y_{(s+1)}\right)=\underline{d_{s}}\left(x_{(s)}\right)+\underline{d_{s}}\left(y_{(s)}\right) \text {. }
$$

Summing up over $s=0,1, \ldots, m-1$ and using that $d_{m}\left(x_{(m)}, y_{(m)}\right)=0$, we get

$$
d(x, y)=d\left(x_{(0)}, y_{(0)}\right)=\sigma^{m}(x)+\sigma^{m}(y) .
$$

To prove (ii), note that $x_{(s)} \neq y_{(s)}$ for all $s \geq 0$. Thus, we have (6.1.5) for all $s \geq 0$. Summing up over $s=0,1, \ldots, n-1$, we obtain for all $n \geq 0$,

$$
d(x, y)=d_{0}\left(x_{(0)}, y_{(0)}\right)=d_{n}\left(x_{(n)}, y_{(n)}\right)+\sigma^{n}(x)+\sigma^{n}(y) .
$$

Therefore all partial sums of the series $\sigma(x)$ and $\sigma(y)$ are bounded above by $d(x, y)$. Hence, both series converge. Taking the limit $n \rightarrow \infty$ in (6.1.6), we obtain (6.1.4).

Lemma 6.2. If $\operatorname{card}\left(X_{\infty}\right) \geq 2$, then the series $\sigma(x)$ converges for all $x \in X$.

This directly follows from Lemma 6.1.(ii) as we can pick $y \in X$ so that $x \nsucceq y$. 
6.2. Proof of Theorem 5.1. Suppose first that $\operatorname{card}\left(X_{\infty}\right)=1$. Then $C$ is (path) connected and $\rho$ is the path pseudometric in $C$. We verify that $\rho\left(x_{(k)}, y_{(k)}\right)=$ $d_{k}\left(x_{(k)}, y_{(k)}\right)$ for all $x, y \in X$ and all $k \geq 0$. The connectedness of $C$ implies that $x \cong y$. Let $m=m(x, y)$ be the meeting index of $x$ and $y$. If $k \geq m$, then $x_{(k)}=y_{(k)}$ and $\rho\left(x_{(k)}, y_{(k)}\right)=d_{k}\left(x_{(k)}, y_{(k)}\right)=0$. If $k<m$, then an injective path from $x_{(k)}$ to $y_{(k)}$ in $C$ is formed by the edges

$$
e_{x_{(k)}}, e_{x_{(k+1)}}, \ldots, e_{x_{(m-1)}}, e_{y_{(m-1)}}, \ldots, e_{y_{(k+1)}}, e_{y_{(k)}} .
$$

Therefore

$$
\rho\left(x_{(k)}, y_{(k)}\right)=\sum_{s=k}^{m-1}\left(\underline{d_{s}}\left(x_{(s)}\right)+\underline{d_{s}}\left(y_{(s)}\right)\right) .
$$

By the definition of the metric $d_{s+1}$, for all $s<m$, we have (6.1.5). Summing up these equalities over $s=k, \ldots, m-1$, we obtain that

$$
d_{k}\left(x_{(k)}, y_{(k)}\right)=d_{k}\left(x_{(k)}, y_{(k)}\right)-d_{m}\left(x_{(m)}, y_{(m)}\right)=\sum_{s=k}^{m-1}\left(\underline{d_{s}}\left(x_{(s)}\right)+\underline{d_{s}}\left(y_{(s)}\right)\right) .
$$

Comparing with (6.2.1), we obtain that $\rho\left(x_{(k)}, y_{(k)}\right)=d_{k}\left(x_{(k)}, y_{(k)}\right)$.

Suppose now that $\operatorname{card}\left(X_{\infty}\right) \geq 2$. By Lemma 6.2 the series (6.1.1) converges for all $x \in X$ and yields a function $\sigma: X \rightarrow \mathbb{R}_{+}$. This function extends to $C$ as follows: for any $a \in C$ lying in the edge $e=e_{x_{(k)}}$ connecting the vertices $x_{(k)}$ and $x_{(k+1)}$ with $x \in X, k \geq 0$, set

$$
\sigma(a)=d_{e}\left(a, x_{(k+1)}\right)+\sum_{s=k+1}^{\infty} \underline{d_{s}}\left(x_{(s)}\right)
$$

where $d_{e}$ is the pseudometric in $e$ induced by the fixed homeomorphism $e \approx$ $\left[0, \underline{d_{k}}\left(x_{(k)}\right)\right]$ if $\underline{d_{k}}\left(x_{(k)}\right)>0$ and $d_{e}=0$ if $\underline{d_{k}}\left(x_{(k)}\right)=0$. The infinite series in the expression for $\bar{\sigma}(a)$ is majorated by $\sigma(x)$ and therefore converges. In particular, $\sigma\left(x_{(k)}\right)=\sum_{s \geq k} \underline{d_{s}}\left(x_{(s)}\right)$ for all $x \in X, k \geq 0$. It is clear that the function $\sigma: C \rightarrow \mathbb{R}_{+}$is continuous. The next lemma implies that $\sigma$ is monotonous in the sense that if $b \in C$ lies below $a \in C$, then $\sigma(b) \leq \sigma(a)$.

Lemma 6.3. If $a, b \in C$ lie in the same component $L$ of $C$, then

$$
|\sigma(a)-\sigma(b)| \leq d_{L}(a, b) \leq \sigma(a)+\sigma(b)
$$

where $d_{L}$ is the path pseudometric in L. Moreover, if $b$ lies below a, then

$$
\sigma(a)=d_{L}(a, b)+\sigma(b) .
$$

Proof. Formula (6.2.3) follows from the definitions. For $a=b$ the formula (6.2.2) is obvious. If one of the points $a, b$ lies below the other one, then (6.2.2) follows from (6.2.3). In all other cases, the injective path from $a$ to $b$ is $\mathrm{V}$-shaped, i.e., goes down from $a$ to a certain vertex $v$ of $L$ and then goes up to $b$ (a path goes up if the inverse path goes down). Then

$$
\sigma(a)=d_{L}(a, v)+\sigma(v), \quad \sigma(b)=d_{L}(b, v)+\sigma(v),
$$

and $d_{L}(a, b)=d_{L}(a, v)+d_{L}(b, v)$. This easily implies (6.2.2). 
To complete the proof of the theorem, consider the map $q: C \rightarrow \pi_{0}(C)=X_{\infty}$ carrying each point to its path connected component. For any $a, b \in C$, set

$$
\rho(a, b)=\left\{\begin{array}{l}
d_{L}(a, b) \quad \text { if } a, b \text { lie in the same component } L \text { of } C, \\
d_{\infty}(q(a), q(b))+\sigma(a)+\sigma(b) \text { if } q(a) \neq q(b) .
\end{array}\right.
$$

We claim that the map $\rho: C \times C \rightarrow \mathbb{R}_{+}$is a pseudometric. That $\rho(a, a)=0$ and $\rho(a, b)=\rho(b, a)$ for all $a, b \in C$ is clear. For points of $C$ lying in the same component $L$, the triangle inequality follows from the one for $d_{L}$. For points of $C$ lying in three different components, the triangle inequality follows from the one for $d_{\infty}$. If $a, b \in L$ for a component $L \subset C$ and $c \in C \backslash L$, then by Lemma 6.3 ,

$$
|\rho(a, c)-\rho(b, c)|=|\sigma(a)-\sigma(b)| \leq d_{L}(a, b)=\rho(a, b)
$$

and

$$
\rho(a, b)=d_{L}(a, b) \leq \sigma(a)+\sigma(b) \leq \rho(a, c)+\rho(b, c) .
$$

We check that $\left.\rho\right|_{X_{k}}=d_{k}$ for all $k$. Pick any $x, y \in X$. If $x \cong y$, then the arguments given in the case of connected $C$ apply and show that $\rho\left(x_{(k)}, y_{(k)}\right)=$ $d_{k}\left(x_{(k)}, y_{(k)}\right)$. If $x \nsucceq y$, then similar arguments show that for all $n \geq k$,

$$
d_{k}\left(x_{(k)}, y_{(k)}\right)=d_{n}\left(x_{(n)}, y_{(n)}\right)+\sum_{s=k}^{n-1}\left(\underline{d_{s}}\left(x_{(s)}\right)+\underline{d_{s}}\left(y_{(s)}\right)\right) .
$$

Taking the limit $n \rightarrow \infty$, we get

$$
d_{k}\left(x_{(k)}, y_{(k)}\right)=d_{\infty}\left(x_{(\infty)}, y_{(\infty)}\right)+\sigma\left(x_{(k)}\right)+\sigma\left(y_{(k)}\right)=\rho\left(x_{(k)}, y_{(k)}\right) .
$$

Finally, we prove that $\rho(a, b) \leq \rho^{\prime}(a, b)$ for any $a, b \in C$ and any pseudometric $\rho^{\prime}$ in $C$ which restricts to $d$ in $X$ and to the path pseudometrics in the components of $C$. It suffices to treat the case where $a, b$ lie in different components, say, $L, M$ of $C$. Pick a path in $L$ starting in $a$, going up, and ending in some $x \in X$. Then $\sigma(x)=d_{L}(x, a)+\sigma(a)$. Similarly, pick a path in $M$ starting in $b$, going up, and ending in some $y \in X$. Then $\sigma(y)=d_{M}(y, b)+\sigma(b)$. We have $q(x)=q(a) \neq$ $q(y)=q(b)$ and therefore

$$
\begin{gathered}
d(x, y)=\rho(x, y)=d_{\infty}(q(x), q(y))+\sigma(x)+\sigma(y) \\
=d_{\infty}(q(a), q(b))+d_{L}(x, a)+\sigma(a)+d_{M}(y, b)+\sigma(b) \\
=\rho(a, b)+d_{L}(x, a)+d_{M}(y, b) .
\end{gathered}
$$

At the same time, the assumptions on $\rho^{\prime}$ imply that

$$
d(x, y)=\rho^{\prime}(x, y) \leq \rho^{\prime}(x, a)+\rho^{\prime}(a, b)+\rho^{\prime}(y, b)=\rho^{\prime}(a, b)+d_{L}(x, a)+d_{M}(y, b) .
$$

We conclude that

$$
\rho(a, b)=d(x, y)-d_{L}(x, a)-d_{M}(y, b) \leq \rho^{\prime}(a, b) .
$$


6.3. Remark. If card $\left(X_{\infty}\right)=1$, then the series $\sigma(x)$ may converge or not. For instance, if in Examples 4.4 and 4.5 we set $\delta_{k}(x)=1$ for all $x \in X, k \geq 0$, then $\sigma^{n}(x)=n$ for all $n$ and $\sigma(x)$ does not converge. Setting $\delta_{k}(x)=2^{-k}$ for all $x, k$, we obtain examples where $\sigma(x)$ converges for all $x$. In general, if $\sigma(x)$ converges for some $x \in X$, then it converges for all $x \in X$. Indeed, the assumption $\operatorname{card}\left(X_{\infty}\right)=1$ ensures that any $x, y \in X$ project to the same element of $X_{k}$ for all sufficiently big $k$ and therefore the series $\sigma(x), \sigma(y)$ differ only in a finite number of terms. Also, if (the only component of) $C$ is special, then all terms of the series $\sigma(x)$ starting from a certain place are equal to zero and $\sigma(x)$ converges.

\section{THE TIGHT SPAN VERSUS THE TRIMMING CYLINDER}

We recall the tight span following $[\mathrm{Dr}$, [DMT] and relate it to the trimming cylinder. We also discuss the tight spans of pseudometric spaces.

7.1. Tight span of a metric space. The tight span of a metric space $(X, d)$ is the metric space $\left(T(X), d_{T}\right)$ consisting of all functions $f: X \rightarrow \mathbb{R}_{+}$such that

$$
f(x)=\sup _{y \in X}(d(x, y)-f(y)) \text { for all } x \in X .
$$

This identity may be restated by saying that

(*) $f(x)+f(y) \geq d(x, y)$ for all $x, y \in X$ and

$(* *)$ for any $x \in X$ and any real number $\varepsilon>0$, there is $y \in X$ such that $f(x)+f(y) \leq d(x, y)+\varepsilon$.

The metric $d_{T}$ in $T(X)$ is defined by

$$
d_{T}(f, g)=\sup _{x \in X}|f(x)-g(x)| \text { for any } f, g \in T(X)
$$

(here the set $\{|f(x)-g(x)|\}_{x \in X}$ is bounded above and has a well-defined supremum). The map carrying any $x \in X$ to the function $X \rightarrow \mathbb{R}_{+}, y \mapsto d(x, y)$ is a metric embedding $X \hookrightarrow T(X)$.

Each $f \in T(X)$ is minimal in the set of functions $f^{\prime}: X \rightarrow \mathbb{R}_{+}$satisfying $(*)$ : if $f \geq f^{\prime}$ (in the sense that $f(x) \geq f^{\prime}(x)$ for all $x \in X$ ), then $f=f^{\prime}$. Indeed,

$$
f(x) \geq f^{\prime}(x) \geq \sup _{y \in X}\left(d(x, y)-f^{\prime}(y)\right) \geq \sup _{y \in X}(d(x, y)-f(y))=f(x)
$$

for all $x \in X$, and so $f(x)=f^{\prime}(x)$.

If $X$ is a singleton, then $T(X)=\{0\}$. If $\operatorname{card}(X) \geq 2$, then (7.1.1) may be reformulated as follows (see $[\mathrm{Dr}]$, Sect. 1.4): for every $x \in X$,

$$
f(x)=\sup _{y \in X \backslash\{x\}}(d(x, y)-f(y)) .
$$

This identity may be restated by saying that

$(*)^{\prime} f(x)+f(y) \geq d(x, y)$ for all distinct $x, y \in X$ and

$(* *)^{\prime}$ for any $x \in X$ and any real number $\varepsilon>0$, there is $y \in X \backslash\{x\}$ such that $f(x)+f(y) \leq d(x, y)+\varepsilon$.

We now relate the tight span $T(X)$ to the trimming cylinder $C(X)$. 
Theorem 7.1. For any metric space $X=(X, d)$, there is a canonical distance preserving map $C(X) \rightarrow T(X)$ extending the standard embedding $X \hookrightarrow T(X)$.

Proof. For $a \in C=C(X)$, define a function $f=f_{a}: X \rightarrow \mathbb{R}_{+}$by $f(x)=\rho(x, a)$ for all $x \in X \subset C$ where $\rho=\rho_{X}$ is the pseudometric in $C$. We claim that $f \in T(X)$. Note that for any $x, y \in X$, the triangle inequality for $\rho$ implies that $f(x)+f(y) \geq \rho(x, y)=d(x, y)$. Thus, $f$ satisfies Condition $(*)$ above. Instead of Condition $(* *)$, we check a stronger claim: for any $x \in X$, there is $y \in X$ such that $f(x)+f(y)=d(x, y)$. In other words, we find $y \in X$ such that $a$ lies between $x$ and $y$ in $C$ (see Section 2.2 for "betweenness"). Assume for concreteness that $a$ lies in an edge of $C$ connecting vertices $v \in X_{k}$ and $p_{k}(v) \in X_{k+1}$ for some $k \geq 0$ (possibly, $a=v$ ). We separate several cases.

(i) Let $x, v$ lie in different components of $C$. Pick $y \in X$ such that $y_{(k)}=v$. The definition of $\rho$ shows that

$$
f(x)+f(y)=\rho(x, a)+\rho(y, a)=\rho(x, a)+\sigma(y)-\sigma(a)=\rho(x, y)=d(x, y) .
$$

(ii) Let $x, v$ lie in the same component of $C$ and $x_{(k)} \neq v$. Pick $y \in X$ such that $y_{(k)}=v$. Then $x \cong y$. Consider the injective path in $C$ going from $y$ down to $y_{(k)}=v$, then further down to $y_{(m)}=x_{(m)}$ where $m=m(x, y)>k$ is the meeting index of $x, y$, and then up to $x$. This path from $y$ to $x$ has length $\rho(x, y)=d(x, y)$. Since the point $a$ lies on this path, its length $d(x, y)$ is equal to $\rho(x, a)+\rho(y, a)=f(x)+f(y)$.

(iii) Let $x_{(k)}=v$ and $\operatorname{card}\left(X_{k}\right) \geq 2$. Pick $y \in X$ such that $x \neq y$. The same argument as in (i) shows that $f(x)+f(y)=d(x, y)$.

(iv) Let $\operatorname{card}\left(X_{k}\right)=1$, i.e., $X_{k}=\{v\}$. Then the edge containing $a$ (i.e., the edge from $v$ to $\left.p_{k}(v)\right)$ has zero length, and $f=\rho(-, a)=\rho(-, v)$. Thus, it is enough to treat the case $a=v$. If $\operatorname{card}\left(X_{k-1}\right) \geq 2$, then there is $y \in X$ such that $x_{(k-1)} \neq y_{(k-1)}$. The injective path from $y$ to $x$ in $C$ must pass through the only element $v$ of $X_{k}$ and so $f(x)+f(y)=d(x, y)$. If $\operatorname{card}\left(X_{k-1}\right)=1$, then $f=\rho(-, u)$ where $u$ is the only element of $X_{k-1}$. Proceeding by induction, we eventually find some $l<k$ such that $\operatorname{card}\left(X_{l}\right) \geq 2$ and the argument above works or deduce that $\operatorname{card}(X)=1$ in which case the theorem is obvious.

We next verify that $d_{T}\left(f_{a}, f_{b}\right)=\rho(a, b)$ for all $a, b \in C$. The case $a=b$ is obvious as both sides are equal to zero. Assume that $a \neq b$ and recall that

$$
d_{T}\left(f_{a}, f_{b}\right)=\sup _{x \in X}\left|f_{a}(x)-f_{b}(x)\right|=\sup _{x \in X}|\rho(x, a)-\rho(x, b)| .
$$

The triangle inequality for $\rho$ yields $d_{T}\left(f_{a}, f_{b}\right) \leq \rho(a, b)$. To prove that this is an equality, we find a point $x \in X \subset C$ such that either $b$ lies between $a$ and $x$ (with respect to the pseudometric $\rho$ in $C$ ) or $a$ lies between $b$ and $x$. In both cases $|\rho(x, a)-\rho(x, b)|=\rho(a, b)$. Note that going up from $a, b$ in $C$ we eventually hit certain elements, respectively, $x, y \in X \subset C$. If $a, b$ belong to different components of $C$, then $a$ lies between $b$ and $x$ as follows from the definition of $\rho$. Assume that $a, b$ belong to the same component $L$ of $C$. If $b$ lies below $a$, then $a$ lies between $b$ and $x$ as follows from the definition of the path pseudometric in $L$. In all other 
cases, an injective path from $y$ to $a$ in $L$ necessarily passes by $b$ and therefore $b$ lies between $a$ and $y$.

Corollary 7.2. For any metric space $X$, there is a canonical metric embedding $\overline{C(X)} \hookrightarrow T(X)$ extending the standard embedding $X \hookrightarrow T(X)$.

The embedding $\bar{C}=\overline{C(X)} \hookrightarrow T(X)$ is induced by the distance preserving map from Theorem [7.1] We will identify $\bar{C}$ with its image in $T(X)$ under this embedding, i.e., view $\bar{C}$ as a metric subspace of $T(X)$.

7.2. The tight span of a pseudometric space. The definition of the tight span via (7.1.1) extends word for word to pseudometric spaces. This however does not give new metric spaces because, by the next lemma, a pseudometric space and its metric quotient have the same tight span.

Lemma 7.3. Let $X=(X, d)$ be a pseudometric space and let $q: X \rightarrow \bar{X}=X / \sim_{d}$ be the projection from $X$ to its metric quotient. Then the formula $h \mapsto h q$, where $h$ runs over $T(\bar{X})$, defines an isometry $T(\bar{X}) \rightarrow T(X)$.

Proof. Let $\bar{d}$ be the metric in $\bar{X}$ induced by $d$. We pick any function $h: \bar{X} \rightarrow \mathbb{R}_{+}$ in $T(\bar{X})$ and verify Conditions $(*),(* *)$ for $h q: X \rightarrow \mathbb{R}_{+}$. For any $x, y \in X$, we have $h q(x)+h q(y) \geq d(x, y)$ because if $q(x)=q(y)$, then $d(x, y)=0$, and if $q(x) \neq q(y)$, then $h q(x)+h q(y) \geq \bar{d}(q(x), q(y))=d(x, y)$. To verify $(* *)$, pick any $x \in X$ and $\varepsilon>0$. Since $h \in T(\bar{X})$ and $q$ is onto, there is $y \in X$ such that

$$
h q(x)+h q(y) \leq \bar{d}(q(x), q(y))+\varepsilon=d(x, y)+\varepsilon .
$$

Thus, $h q \in T(X)$. That the map $T(\bar{X}) \rightarrow T(X), h \mapsto h q$ is injective and metric preserving is clear from the definitions. To prove surjectivity, we show that each function $f \in T(X)$ takes equal values on $\sim_{d^{-}}$equivalent points of $X$. We have $f(y)-d(x, y) \leq f(x)$ for all $x, y \in X$ because (cf. [DMT, Section 2)

$$
\begin{gathered}
f(y)-d(x, y)=\sup _{z \in X}(d(y, z)-f(z))-d(x, y) \\
=\sup _{z \in X}(d(y, z)-f(z)-d(x, y)) \leq \sup _{z \in X}(d(y, x)+d(x, z)-f(z)-d(x, y)) \\
=\sup _{z \in X}(d(x, z)-f(z))=f(x) .
\end{gathered}
$$

If $d(x, y)=0$, then we get $f(y) \leq f(x)$. Exchanging $x, y$, we get $f(x) \leq f(y)$. Thus, if $d(x, y)=0$, then $f(x)=f(y)$. As a consequence, $f=h q$ for a function $h: \bar{X} \rightarrow \mathbb{R}_{+}$. Conditions $(*),(* *)$ for $f$ imply the same conditions for $h$.

\section{TRIMMING VERSUS THE TIGHT SPAN}

We discuss further relations between trimming and the tight span. In this section, $X=(X, d)$ is a metric space. 
8.1. The canonical embedding. Recall the function $\underline{d}: X \rightarrow \mathbb{R}_{+}$induced by $d$.

Theorem 8.1. There is a canonical metric embedding $T(t(X)) \hookrightarrow T(X)$ whose image consists of all $f \in T(X)$ such that $f \geq \underline{d}$.

Proof. Let $t(X)=\left(X_{1}, d_{1}\right)$ as in Section 3.2. Assume first that $X_{1}$ is a singleton. Then $T\left(X_{1}\right)=\{0\}$ and the embedding $T\left(X_{1}\right) \hookrightarrow T(X)$ carries 0 to $\underline{d}$. We need to prove that $\underline{d} \in T(X)$. If $\operatorname{card}(X)=1$, then $\underline{d}=0 \in T(X)$. If $\operatorname{card}(X) \geq 2$, then our assumption on $X_{1}$ implies that $d(x, y)=\underline{d}(x)+\underline{d}(y)$ for all distinct $x, y \in X$. Applying (7.1.3) to $f=\underline{d}$, we deduce that $\underline{d} \in T(X)$. By the minimality of any $f \in T(X)$, if $f \geq \underline{d}$, then $f=\underline{d}$. This gives the second claim of the theorem.

Assume now that $\operatorname{card}\left(X_{1}\right) \geq 2$, and let $p: X \rightarrow X_{1}$ be the trimming projection. Given $g \in T\left(X_{1}\right)$, we define a function $\hat{g}: X \rightarrow \mathbb{R}_{+}$by

$$
\hat{g}(x)=g p(x)+\underline{d}(x)=g\left(x_{(1)}\right)+\underline{d}(x)
$$

for all $x \in X$. We claim that $\hat{g} \in T(X)$. To see it, we check Conditions $(*)^{\prime}$ and $(* *)^{\prime}$ of Section 7.1 for $f=\hat{g}$. To check $(*)^{\prime}$, we separate two cases: $p(x)=p(y)$ and $p(x) \neq p(y)$. If $p(x)=p(y)$, then $(*)^{\prime}$ holds because

$$
\hat{g}(x)+\hat{g}(y) \geq \underline{d}(x)+\underline{d}(y)=d(x, y) .
$$

If $p(x) \neq p(y)$, then $(*)^{\prime}$ holds because

$$
\begin{gathered}
\hat{g}(x)+\hat{g}(y)=g p(x)+g p(y)+\underline{d}(x)+\underline{d}(y) \\
\geq d_{1}(p(x), p(y))+\underline{d}(x)+\underline{d}(y)=d(x, y)
\end{gathered}
$$

where the inequality follows from the inclusion $g \in T\left(X_{1}\right)$ and the final equality holds by the definition of $d_{1}$. To verify $(* *)^{\prime}$, pick any $x \in X$ and $\varepsilon>0$. Using $(* *)^{\prime}$ for $g \in T\left(X_{1}\right)$ and using the surjectivity of $p: X \rightarrow X_{1}$, we obtain an element $y \in X$ such that $p(x) \neq p(y)$ and

$$
d_{1}(p(x), p(y))+\varepsilon \geq g p(x)+g p(y) .
$$

Then $x \neq y$ and $(* *)^{\prime}$ for $\hat{g}$ follows:

$$
\begin{aligned}
& d(x, y)+\varepsilon=d_{1}(p(x), p(y))+\underline{d}(x)+\underline{d}(y)+\varepsilon \\
& \geq g p(x)+g p(y)+\underline{d}(x)+\underline{d}(y)=\hat{g}(x)+\hat{g}(y) .
\end{aligned}
$$

The map $T\left(X_{1}\right) \rightarrow T(X), g \mapsto \hat{g}$ is a metric embedding: for any $g, h \in T\left(X_{1}\right)$,

$$
\begin{aligned}
d_{T}(\hat{g}, \hat{h}) & =\sup _{x \in X}|\hat{g}(x)-\hat{h}(x)|=\sup _{x \in X}|g p(x)-h p(x)| \\
& =\sup _{z \in X_{1}}|g(z)-h(z)|=\left(d_{1}\right)_{T}(g, h) .
\end{aligned}
$$

To show that any $f \in T(X)$ satisfying $f \geq \underline{d}$ lies in the image of our embedding, set $f_{-}=f-\underline{d}: X \rightarrow \mathbb{R}_{+}$and recall the pseudometric $d^{-}$in $X$ defined in Section 3.2 Since $\operatorname{card}(X) \geq \operatorname{card}\left(X_{1}\right) \geq 2$, we have by (7.1.3) that for any $x \in X$,

$$
\begin{aligned}
f_{-}(x) & =f(x)-\underline{d}(x)=\sup _{y \in X \backslash\{x\}}(d(x, y)-\underline{d}(x)-\underline{d}(y)+\underline{d}(y)-f(y)) \\
& =\sup _{y \in X \backslash\{x\}}\left(d^{-}(x, y)-f_{-}(y)\right)=\sup _{y \in X}\left(d^{-}(x, y)-f_{-}(y)\right) .
\end{aligned}
$$


The last equality holds because its left-hand side is non-negative (being equal to $\left.f_{-}(x)\right)$ while $d^{-}(x, x)-f_{-}(x)=-f_{-}(x) \leq 0$. Thus, $f_{-} \in T\left(X, d^{-}\right)$. By Lemma 7.3. $f_{-}=g p$ for some $g \in T\left(X_{1}\right)$. Then $f=f_{-}+\underline{d}=\hat{g}$.

8.2. The trimming filtration. From now on, we view $T(t(X))=T\left(X_{1}\right)$ as a metric subspace of $T(X)$ via the embedding from Theorem 8.1 Iterating the inclusion $T(X) \supset T\left(X_{1}\right)$, we obtain a filtration

$$
T(X) \supset T\left(X_{1}\right) \supset T\left(X_{2}\right) \supset T\left(X_{3}\right) \supset \cdots
$$

where $t^{n}(X)=\left(X_{n}, d_{n}\right)$ for all $n \geq 1$. In 8.2.1), we identify each $T\left(X_{n}\right)$ with its image under the metric embedding $T\left(X_{n}\right) \hookrightarrow T(X)$ obtained as the composition of the embeddings $T\left(X_{n}\right) \hookrightarrow T\left(X_{n-1}\right) \hookrightarrow \cdots \hookrightarrow T(X)$. This composition carries any $f \in T\left(X_{n}\right)$ to the function $\hat{f}: X \rightarrow \mathbb{R}_{+}$defined by $\hat{f}(x)=f\left(x_{(n)}\right)+\sigma^{n}(x)$ for $x \in X$. Clearly, $\hat{f} \geq \sigma^{n}$. An induction on $n$ deduces from Theorem 8.1 that

$$
T\left(X_{n}\right)=\left\{g \in T(X) \mid g \geq \sigma^{n}\right\} \subset T(X) .
$$

Theorem 8.2. All terms of the filtration (8.2.1) are closed subsets of $T(X)$.

Proof. It suffices to show that the set $T\left(X_{1}\right) \subset T(X)$ is closed in $T(X)$ and to apply induction. We prove that the complementary set $U=T(X) \backslash T\left(X_{1}\right)$ is open in $T(X)$. By Theorem 8.1, for any $f \in U$, there is $a \in X$ such that $0 \leq f(a)<\underline{d}(a)$. We claim that the open ball $B \subset T(X)$ with center $f$ and radius $r=\underline{d}(a)-f(a)>0$ is contained in $U$. Indeed, if $g \in B$, then

$$
r>d_{T}(f, g)=\sup _{x \in X}|f(x)-g(x)| \geq g(a)-f(a) .
$$

Therefore $g(a)<f(a)+r=\underline{d}(a)$. Thus, $g \nsupseteq \underline{d}$ and so $g \in U$. We conclude that $U$ is open and $T\left(X_{1}\right)$ is closed.

\subsection{The metric space $\tau$. Consider the set}

$$
\tau=\tau(X)=\cap_{n \geq 1} T\left(X_{n}\right) \subset T(X)
$$

and endow it with the metric obtained by restricting the one in $T(X)$.

Theorem 8.3. If either $\operatorname{card}\left(X_{\infty}\right) \geq 2$ or $\operatorname{card}\left(X_{\infty}\right)=1$ and the series $\sigma(x)$ converges for all $x \in X$, then there is an isometry $\tau \approx T\left(X_{\infty}\right)$. If $\operatorname{card}\left(X_{\infty}\right)=1$ and the series $\sigma(x)$ does not converge for some $x \in X$, then $\tau=\emptyset$.

Proof. Recall that $X_{\infty}=X / \cong$ where $\cong$ is the equivalence relation in $X$ defined in Section 3.2. We start with the case $\operatorname{card}\left(X_{\infty}\right) \geq 2$. By Lemma 6.2, the series $\sigma(x)$ converges for all $x \in X$ and yields a function $\sigma: X \rightarrow \mathbb{R}_{+}$. For any function $f: X_{\infty} \rightarrow \mathbb{R}_{+}$, we define a function $\tilde{f}: X \rightarrow \mathbb{R}_{+}$by $\tilde{f}(x)=f\left(x_{(\infty)}\right)+\sigma(x)$. We claim that if $f \in T\left(X_{\infty}\right)$, then $\tilde{f} \in T(X)$. Note that $\operatorname{card}(X) \geq \operatorname{card}\left(X_{\infty}\right) \geq 2$. Therefore to verify the inclusion $\tilde{f} \in T(X)$, it suffices to verify for $\tilde{f}$ Conditions $(*)^{\prime}$ and $(* *)^{\prime}$ from Section 7.1 . To verify $(*)^{\prime}$, pick any distinct $x, y \in X$. If $x \cong y$, then Formula (6.1.3) implies that

$$
d(x, y) \leq \sigma(x)+\sigma(y) \leq \tilde{f}(x)+\tilde{f}(y) .
$$


If $x \not y y$, then $x_{(\infty)} \neq y_{(\infty)}$. Formula (6.1.4) and Condition $(*)^{\prime}$ on $f$ give

$$
d(x, y) \leq f\left(x_{(\infty)}\right)+f\left(y_{(\infty)}\right)+\sigma(x)+\sigma(y)=\tilde{f}(x)+\tilde{f}(y) .
$$

To verify $(* *)^{\prime}$, pick any $x \in X$ and $\varepsilon>0$. Since $f: X_{\infty} \rightarrow \mathbb{R}_{+}$satisfies $(* *)^{\prime}$ and the projection $X \rightarrow X_{\infty}$ is onto, there is $y \in X$ such that $x_{(\infty)} \neq y_{(\infty)}$ and

$$
f\left(x_{(\infty)}\right)+f\left(y_{(\infty)}\right) \leq d_{\infty}\left(x_{(\infty)}, y_{(\infty)}\right)+\varepsilon .
$$

Then $x \neq y$ and by (6.1.4),

$$
\tilde{f}(x)+\tilde{f}(y) \leq d_{\infty}\left(x_{(\infty)}, y_{(\infty)}\right)+\varepsilon+\sigma(x)+\sigma(y)=d(x, y)+\varepsilon .
$$

Thus, $\tilde{f} \in T(X)$. The map $T\left(X_{\infty}\right) \rightarrow T(X), f \mapsto \tilde{f}$ is a metric embedding:

$$
\begin{aligned}
d_{T}\left(\tilde{f}_{1}, \tilde{f}_{2}\right) & =\sup _{x \in X}\left|\tilde{f}_{1}(x)-\tilde{f}_{2}(x)\right|=\sup _{x \in X}\left|f_{1}\left(x_{(\infty)}\right)-f_{2}\left(x_{(\infty)}\right)\right| \\
& =\sup _{z \in X_{\infty}}\left|f_{1}(z)-f_{2}(z)\right|=\left(d_{\infty}\right)_{T}\left(f_{1}, f_{2}\right)
\end{aligned}
$$

for any $f_{1}, f_{2} \in T\left(X_{\infty}\right)$. Also, for all $n \geq 1$, the obvious inequalities $\tilde{f} \geq \sigma \geq \sigma^{n}$ imply that $\tilde{f} \in T\left(X_{n}\right) \subset T(X)$. So, $\tilde{f} \in \tau$.

It remains to show that for each $g \in \tau$ there is $f \in T\left(X_{\infty}\right)$ such that $g=\tilde{f}$. By Theorem 8.1, the function $g-\sigma^{1}=g-\underline{d}$ is the composition of the projection $X \rightarrow t(X)$ and a certain function $g_{1} \in T\left(X_{1}\right)$. Proceeding by induction, we deduce that for each $n \geq 1$, the function $g-\sigma^{n}$ is the composition of the projection $X \rightarrow X_{n}$ and a function $g_{n} \in T\left(X_{n}\right)$. Thus, $g \geq \sigma^{n}$ for all $n \geq 1$ and therefore $g \geq \sigma$. We check now that the function $g-\sigma \geq 0$ is the composition of the projection $X \rightarrow X_{\infty}$ and a function $X_{\infty} \rightarrow \mathbb{R}_{+}$. If two points $x, y \in X$ project to the same point of $X_{\infty}$, then there is an integer $n \geq 0$ such that $x_{(n)}=y_{(n)}$. Then

$$
\left(g-\sigma^{n}\right)(x)=g_{n}\left(x_{(n)}\right)=g_{n}\left(y_{(n)}\right)=\left(g-\sigma^{n}\right)(y) .
$$

Also, $x_{(s)}=y_{(s)}$ for all $s \geq n$ and so

$$
\left(\sigma-\sigma^{n}\right)(x)=\sum_{s \geq n} \underline{d_{s}}\left(x_{(s)}\right)=\sum_{s \geq n} \underline{d_{s}}\left(y_{(s)}\right)=\left(\sigma-\sigma^{n}\right)(y) .
$$

Therefore, $(g-\sigma)(x)=(g-\sigma)(y)$. Thus, the function $g-\sigma \geq 0$ is the composition of the projection $X \rightarrow X_{\infty}$ and a function $f: X_{\infty} \rightarrow \mathbb{R}_{+}$. So, $g=\tilde{f}$. We claim that $f \in T\left(X_{\infty}\right)$. To see it, we verify that $f$ satisfies Conditions $(*)$ and $(* *)$ from Section 7.1. To verify $(*)$, pick any $x, y \in X$. If $x_{(\infty)} \neq y_{(\infty)}$, then

$$
\begin{gathered}
f\left(x_{(\infty)}\right)+f\left(y_{(\infty)}\right)=g(x)-\sigma(x)+g(y)-\sigma(y) \\
\geq d(x, y)-\sigma(x)-\sigma(y)=d_{\infty}\left(x_{(\infty)}, y_{(\infty)}\right)
\end{gathered}
$$

where the inequality follows from the assumption $g \in T(X)$ and the last equality holds by (6.1.4). If $x_{(\infty)}=y_{(\infty)}$, then

$$
f\left(x_{(\infty)}\right)+f\left(y_{(\infty)}\right) \geq 0=d_{\infty}\left(x_{(\infty)}, y_{(\infty)}\right) .
$$

To verify (**), pick any $x \in X$ and $\varepsilon>0$. Since $g \in T(X)$, there is $y \in X$ such that $g(x)+g(y) \leq d(x, y)+\varepsilon$. Then

$$
f\left(x_{(\infty)}\right)+f\left(y_{(\infty)}\right)=g(x)-\sigma(x)+g(y)-\sigma(y) \leq d(x, y)+\varepsilon-\sigma(x)-\sigma(y) .
$$


If $x_{(\infty)} \neq y_{(\infty)}$, then the right-hand side is equal to $d_{\infty}\left(x_{(\infty)}, y_{(\infty)}\right)+\varepsilon$ and we are done. If $x_{(\infty)}=y_{(\infty)}$, then the right-hand side is smaller than or equal to $\varepsilon=d_{\infty}\left(x_{(\infty)}, y_{(\infty)}\right)+\varepsilon$ as follows from (8.3.1).

Assume that $\operatorname{card}\left(X_{\infty}\right)=1$, i.e., that $X_{\infty}$ is a singleton. If $\tau \neq \emptyset$, then any $f \in \tau=\cap_{n \geq 1} T^{n}(X)$ majorates all the functions $\left\{\sigma^{n}\right\}_{n}$ on $X$. Therefore the series $\sigma(x)$ converges for all $x \in X$. This implies the last claim of the theorem. Suppose now that the series $\sigma(x)$ converges for all $x \in X$. It defines then a function $\sigma: X \rightarrow \mathbb{R}_{+}$. Below we prove that $\sigma \in T(X)$. This will imply the claim of the theorem. Indeed, since $\sigma \geq \sigma^{n}$ for all $n \geq 1$, we have then $\sigma \in \tau$. Any $f \in \tau$ satisfies $f \geq \sigma^{n}$ for all $n \geq 1$ and so $f \geq \sigma$. By the minimality of $f \in T(X)$ (see Section 7.10), the inequality $f \geq \sigma$ implies that $f=\sigma$. Thus, $\tau=\{\sigma\}$ is isometric to $T\left(X_{\infty}\right)=\{0\}$. To prove the inclusion $\sigma \in T(X)$, we verify Conditions $(*)$ and (**) from Section 7.1 The distance $d(x, y)$ between any points $x, y \in X$ may be computed from the equality $d(x, y)=\rho(x, y)$, where $\rho$ is the pseudometric in the trimming cylinder of $X$, and the expression (6.2.1) for $\rho(x, y)$ where $k=0$ and $m=m(x, y)$. This gives

$$
d(x, y)=\sigma^{m}(x)+\sigma^{m}(y) \leq \sigma(x)+\sigma(y)
$$

which is Condition $(*)$ for $\sigma$. To check $(* *)$ for $\sigma$, pick any $x \in X$ and $\varepsilon>0$. The assumption $\operatorname{card}\left(X_{\infty}\right)=1$ ensures that for every $y \in X$, there is an integer $k \geq 0$ such that $x_{(k)}=y_{(k)}$. Let $k_{y}$ be the smallest such integer. If the set of integers $\left\{k_{y} \geq 0\right\}_{y \in X}$ is finite, then either $\operatorname{card}(X)=1$ or there is an integer $K \geq 1$ such that $\operatorname{card}\left(X_{K-1}\right) \geq 2$ and $\operatorname{card}\left(X_{K}\right)=1$. If $\operatorname{card}(X)=1$, then $\sigma=0 \in T(X)=\{0\}$. If $\operatorname{card}\left(X_{K-1}\right) \geq 2$ and $\operatorname{card}\left(X_{K}\right)=1$, then $\sigma=\sigma^{K}$ and there is $y \in X \backslash\{x\}$, such that $y_{(K-1)} \neq x_{(K-1)}$ and $y_{(K)}=x_{(K)}$. Then

$$
\sigma(x)+\sigma(y)=\sigma^{K}(x)+\sigma^{K}(y)=\rho(x, y)=d(x, y) \leq d(x, y)+\varepsilon .
$$

If the set $\left\{k_{y}\right\}_{y \in X}$ is infinite, then we can find $y \in X \backslash\{x\}$ with $k_{y}$ so big that $\sigma(x)-\sigma^{k_{y}}(x)<\varepsilon / 2$. The equality $y_{\left(k_{y}\right)}=x_{\left(k_{y}\right)}$ implies that

$$
\sigma(y)-\sigma^{k_{y}}(y)=\sigma(x)-\sigma^{k_{y}}(x)<\varepsilon / 2 .
$$

Then

$$
\sigma(x)+\sigma(y)<\sigma^{k_{y}}(y)+\sigma^{k_{y}}(x)+\varepsilon=\rho(x, y)+\varepsilon=d(x, y)+\varepsilon .
$$

Here the equality in the middle follows from the definition of the path pseudometric $\rho$ and the conditions $y_{\left(k_{y}-1\right)} \neq x_{\left(k_{y}-1\right)}, y_{\left(k_{y}\right)}=x_{\left(k_{y}\right)}$. Thus, Condition (**) also holds for $\sigma$. So, $\sigma \in T(X)$.

\section{MAIN THEOREM}

9.1. Main theorem. We state our main result on the tight span $T(X)$ of a metric space $X=(X, d)$. Recall the subspaces $\bar{C}$ and $\tau$ of $T(X)$. Recall the special components of the trimming cylinder $C=(C, \rho)$ and the roots of their projections to $\bar{C}$. The set of these roots is denoted $\bar{C}$.

Theorem 9.1. For any metric space $X=(X, d)$,

$$
T(X)=\tau \cup \bar{C} \quad \text { and } \quad \tau \cap \bar{C}=\bar{C} \bullet
$$


Proof. We first consider the case where $\operatorname{card}\left(X_{\infty}\right) \geq 2$. Consider the distance preserving map $C \rightarrow T(X), a \mapsto f_{a}$ from Theorem 7.1 To prove the equality $T(X)=\tau \cup \bar{C}$, it suffices to show that each function $f: X \rightarrow \mathbb{R}_{+}$belonging to $T(X) \backslash \tau$ is equal to $f_{a}$ for some $a \in C$. We prove a stronger claim: $f=f_{a}$ for some $a \in C$ such that $\sigma(a)>0$ where $\sigma: C \rightarrow \mathbb{R}_{+}$is the function defined in the proof of Theorem [5.1. Suppose first that $f \notin T\left(X_{1}\right)$. By Theorem 8.1, there is $y \in X$ such that $0 \leq f(y)<\underline{d}(y)$. The edge of $C$ connecting $y=y_{(0)}$ to $y_{(1)}$ has length $\underline{d}(y)$ and contains a (unique) point $a$ such that $\rho(y, a)=f(y)$. Then

$$
\sigma(a) \geq \rho\left(a, y_{(1)}\right)=\underline{d}(y)-f(y)>0
$$

and $f_{a}(y)=\rho(y, a)=f(y)$. For any $x \in X \backslash\{y\}$, Condition $(*)$ in Section 7.1 and the fact that $a$ lies between $x$ and $y$ in $C$ imply that

$$
f(x) \geq d(y, x)-f(y)=\rho(y, x)-\rho(y, a)=\rho(x, a)=f_{a}(x) .
$$

Thus, $f \geq f_{a}$. Since $f, f_{a} \in T(X)$, we conclude that $f=f_{a}$.

Suppose now that $f \in T\left(X_{n}\right) \backslash T\left(X_{n+1}\right)$ with $n \geq 1$. By Section 8.2, the embedding $T\left(X_{n}\right) \hookrightarrow T(X)$ carries any function $g: X_{n} \rightarrow \mathbb{R}_{+}$in $T\left(X_{n}\right)$ to the function $\hat{g}: X \rightarrow \mathbb{R}_{+}$defined by $\hat{g}(x)=g\left(x_{(n)}\right)+\sigma^{n}(x)$ for all $x \in X$. Since $f \notin T\left(X_{n+1}\right)=T\left(t\left(X_{n}\right)\right) \subset T\left(X_{n}\right)$, Theorem 8.1 implies that there is $y \in X_{n}$ such that $0 \leq f(y)<\underline{d_{n}}(y)$. The edge of $C$ connecting $y \in X_{n}$ to the projection of $y$ to $X_{n+1}$ has length $d_{n}(y)$ and contains a (unique) point $a$ such that $\rho(y, a)=f(y)$. As above, $\sigma(a) \geq \underline{d}_{n}(y)-f(y)>0$. For any $x \in X$ such that $x_{(n)}=y$, the point $y$ lies between $x$ and $a$ in $C$, and therefore

$$
\hat{f}(x)=f(y)+\sigma^{n}(x)=\rho(y, a)+\rho(x, y)=\rho(x, a)=f_{a}(x) .
$$

For any $x \in X$ such that $x_{(n)} \neq y$, the point $a$ lies between $x$ and $y$ in $C$. The inclusion $f \in T\left(X_{n}\right)$ and Condition $(*)$ of Section 7.1 yield

$$
\begin{gathered}
\hat{f}(x)=f\left(x_{(n)}\right)+\sigma^{n}(x) \geq d_{n}\left(x_{(n)}, y\right)-f(y)+\sigma^{n}(x)= \\
=\rho\left(x_{(n)}, y\right)-\rho(y, a)+\rho\left(x, x_{(n)}\right) \geq \rho(x, y)-\rho(y, a)=\rho(x, a)=f_{a}(x) .
\end{gathered}
$$

So, $\hat{f} \geq f_{a}$ and, since $\hat{f}, f_{a} \in T(X)$, we have $\hat{f}=f_{a}$.

The arguments above prove the inclusion $T(X) \backslash \tau \subset \bar{C} \backslash \bar{C}$. As a consequence, $T(X)=\tau \cup \bar{C}$ and $\bar{C}_{\bullet} \subset \tau$. It remains only to show that $\tau \cap(\bar{C} \backslash \bar{C} \bullet)=\emptyset$, i.e., that $f_{a} \notin \tau$ for any $a \in C$ such that $\sigma(a)>0$. Assume for concreteness that $a=x_{(k)}$ or $a$ lies inside the edge of $C$ connecting the vertices $x_{(k)}, x_{(k+1)}$ where $x \in X, k \geq$ 0 . Let $L \subset C$ be the component of $C$ containing $x$ and $a$. The condition $\sigma(a)>0$ implies that there is $n \geq k$ such that $d_{n}\left(x_{(n)}\right)>0$. The definition of the path pseudometric $d_{L}$ in $L$ implies that $d_{L}\left(\overline{\left.a, x_{(n+1)}\right)} \geq \underline{d}_{n}\left(x_{(n)}\right)>0\right.$. It is also clear that $a$ lies between the points $x$ and $x_{(n+1)}$ of $L$. Then

$\sigma^{n+1}(x)=d_{L}\left(x, x_{(n+1)}\right)=d_{L}(x, a)+d_{L}\left(a, x_{(n+1)}\right)>d_{L}(x, a)=\rho(x, a)=f_{a}(x)$.

We conclude that the function $f_{a}$ does not majorate the function $\sigma^{n+1}: X \rightarrow \mathbb{R}_{+}$. By Section 8.2 $f_{a} \notin T\left(X_{n+1}\right) \subset T(X)$. Therefore, $f_{a} \notin \tau$.

Suppose now that $\operatorname{card}\left(X_{\infty}\right)=1$ so that $C$ is (path) connected. If the series $\sigma(x)$ converges for all $x \in X$ (and defines a function $\sigma: X \rightarrow \mathbb{R}_{+}$), then the 
theorem is proved exactly as in the case $\operatorname{card}\left(X_{\infty}\right) \geq 2$. If the series $\sigma(x)$ does not converge for some $x \in X$, then $C$ is non-special and $\bar{C}_{\bullet}=\emptyset$. The equality $T(X)=\tau \cup \bar{C}$ is proved then as in the case $\operatorname{card}\left(X_{\infty}\right) \geq 2$ suppressing all references to $\bar{C}_{\bullet}, \sigma$. Also, $\tau=\emptyset$ by Theorem 8.3 and so $\tau \cap \bar{C}=\emptyset=\bar{C}_{\bullet}$.

9.2. Remarks. 1. When $C$ is (path) connected, i.e., $\operatorname{card}\left(X_{\infty}\right)=1$, we can explicitly compute the sets $\tau$ and $\bar{C}$. . We consider three cases:

(i) (the only component of) $C$ is special, or

(ii) $C$ is not special but the series $\sigma(x)$ converges for all $x \in X$ and defines a function $\sigma: X \rightarrow \mathbb{R}_{+}$, or

(iii) the series $\sigma(x)$ does not converge for some $x \in X$.

In the cases (i) and (ii), the proof of Theorem 8.3 shows that $\tau=\{\sigma\}$. In the case (iii), the same theorem gives $\tau=\emptyset$. The set $\bar{C}_{\bullet}$ is computed from the definitions using the connectedness of $C$. Namely, $\bar{C} \bullet=\{\sigma\}$ in the case (i) and $\bar{C}_{\bullet}=\emptyset$ in the cases (ii) and (iii).

2. For $x \in X$, let $L_{x}$ be the connected component of the trimming cylinder $C=C(X)$ containing $x \in X=X_{0} \subset C$. If the pseudometric tree $L_{x}$ is special, then the root of its metric quotient $\overline{L_{x}}$ belongs to the set $\bar{C} \bullet \subset \tau$. Comparing the canonical embedding $\bar{C} \hookrightarrow T(X)$ with the isometry $T\left(X_{\infty}\right)=\tau$, one can check that the root in question is the point $x_{(\infty)} \in X_{\infty} \subset T\left(X_{\infty}\right)=\tau$.

\section{REFERENCES}

[BCK] A. H. Bilge, D. Çelik, Ş. Koçak, An equivalence class decomposition of finite metric spaces via Gromov products. Discrete Mathematics 340:8 (2017), 1928-1932.

[Dr] A. Dress, Trees, tight extensions of metric spaces, and the cohomological dimension of certain groups: a note on combinatorial properties of metric spaces. Adv. in Math. 53 (1984), 321402.

[DMT] A. Dress, V. Moulton, W. Terhalle, T-theory: an overview. European J. Combin. 17 (1996), 161175.

[Is] J. R. Isbell, Six theorems about injective metric spaces. Comment. Math. Helv. 39 (1964), 6576.

[Me] K. Menger, Untersuchungen uber allgemeine metrik, Math. Ann. 100 (1928), 75-163.

[Tu] V. Turaev, Trimming of finite metric spaces. arXiv:1612.06760.

Department of Mathematics,

INDIANA UNIVERSITY

BLOOMINGTON IN47405

USA

E-MAIL:VTOURAEV@INDIANA.EDU 